\title{
Neuroimaging in Craniocerebral Trauma
}

Rajul Rastogi ${ }^{*}$, Sumeet Bhargava, Pawan Joon, Yuktika Gupta, Asif Majid Wani and Vijai Pratap

Teerthanker Mahaveer Medical College and Research Center, Moradabad, UP, INDIA

*Corresponding author: Rajul Rastogi, Assistant Professor, Department of Radiodiagnosis, Teerthanker Mahaveer Medical College and Research Center, Moradabad, Uttar Pradesh, India, Tel: +91-9319942162; E-mail: eesharastogi@gmail.com

Rec date: May 02, 2016; Acc date: Jul 02, 2016; Pub date: Jul 04, 2016

Copyright: (c) 2016 Rastogi R, et al. This is an open-access article distributed under the terms of the Creative Commons Attribution License, which permits unrestricted use, distribution, and reproduction in any medium, provided the original author and source are credited.

\begin{abstract}
In the present era, trauma is the commonest cause of death in the middle aged group. Cranio Cerebral Trauma (CCT) is common in both urban and rural areas and is associated with high morbidity and mortality rates. In any case of trauma, the outcome depends on two major issues: detection of type and extent of injury and the time taken for management. Though, the presenting neurologic status of the patient is the single best predictor of clinical outcome, but imaging determines the prognosis of these patients especially when the Glasgow coma scale (GCS) is high. There have been major advancements in the field of neuroimaging for CCT over the last decade.
\end{abstract}

Keywords: Neuroimaging; Craniocerebral; Trauma

\section{Introduction}

In the present era, trauma is the commonest cause of death in the middle aged group [1]. Cranio cerebral trauma (CCT) is common in both urban and rural areas and is associated with high morbidity and mortality rates. In any case of trauma, the outcome depends on two major issues: detection of type and extent of injury and the time taken for management. Though, the presenting neurologic status of the patient is the single best predictor of clinical outcome, but imaging determines the prognosis of these patients especially when the Glasgow coma scale (GCS) is high. There have been major advancements in the field of neuroimaging for CCT over the last decade. Computed tomography (CT) especially non enhanced scan (NECT) plays a major role in the evaluation of CCT with magnetic resonance imaging (MRI) (including MR spectroscopy and functional imaging) serving primarily as a problem-solvng tool in cases with discordance between clinical state of patient image NECT scan; cases with high suspicion of diffuse axonal injury image in chronic cases with traumatic sequelae where NECT is equivocal or inconclusive.

Though, positron emission tomography (PET) and single photon emission computed tomography (SPECT) have yielded new concepts in to the understanding of CCT yet it is reserved for difficult cases and research purposes. Hence, this article discusses the neuroimaging in CCT patients with NECT scans as prime focus. But before we proceed, let us first understand the role of CT and MRI in neuroimaging.

CT has a higher spatial resolution than MRI and with the advent of multislice scanners; the time of examination is reduced to a few seconds. Multislice scanners provide thinner scans with excellent multiplanar reconstruction. Because of faster scanning, image degradation by motion in unconscious and irritable patients is minimal. CT is the most sensitive modality for accurate detection of skull fractures. Hence, CT is the first investigation of choice in acute CCT.

MRI has a better soft tissue resolution and is more sensitive in detecting parenchymal lesions of brain especially in the white matter and non-hemorrhagic lesions. It can detect early hemorrhage and can also stage it. Lesions close to bone are more accurately detected by MRI. MRI is free from ionizing radiations and beam hardening artefacts, which are major disadvantages of CT imaging in CCT. Major disadvantages of MRI include longer examination times, high susceptibility to motion, incompatibility with life support systems, limited accessibility and higher cost.

Hence, MRI is indicated in subacute or chronic; relatively stable CCT patients with clinical findings unexplainable by CT findings. $3 \mathrm{~T}$ MRI systems overcome some of the disadvantages of $1.5 \mathrm{~T}$ MRI systems by decreasing acquisition time, increasing spatial resolution and improved MRS, DWI and fMRI. However, it still remains a problem solving tool because of its limited availability and high cost.

Indications of Imaging in Craniocerebral Trauma

\section{Absolute [2]}

- GCS of less than 8 or fall of greater than 3

- Unconsciousness lasting more than 5 minutes

- Depressed skull fracture

- Penetrating / perforating injury

- Neurological deficit

- Pupillary inequality

- Amnesia

- Hemorrhagic disorder

- Patients on anticoagulant therapy

\section{Relative [3]}

- Age more than 60 years

- Persistent headache

- Persistent vomiting especially bloody

- Seizures

- Concomitant alcohol or drug intoxication

- Memory deficit

- Evidence of trauma above clavicles 


\section{Classification of craniocerebral injuries $[4,5]$}

- On the basis of location: Intra-axial (parenchymal) and extra-axial (extraparenchymal-subdural/extradural/intraventricular)

- On the basis of mechanism: Penetrating/open and closed/blunt

- On the basis of timing: Primary (present at the time of examination immediately after trauma/injury occurring as a direct effect of trauma e.g. contusions, diffuse axonal injury, nerve injury, fractures) and secondary (a consequence of primary trauma occurring during the course of time e.g. vasospasm, infarcts, edema, herniation)

- On the basis of clinical severity: Mild (GCS: >13), Moderate (GCS: 9-12) and severe (GCS: 3-8).

\section{Imaging Options in CCT}

\section{Non-contrast CT}

It is the investigation of choice in acute trauma settings. It detects nearly all the primary lesions and can be used to simultaneously image the face and spine. Images should be examined in brain and bone window settings and if required, at intermediate settings to visualize hematoma, air and fracture better. Acute hematoma appears hyperdense to gray matter.

Presence of hypodense areas within the hyperdense hematoma of arterial origin is indicative of active bleeding and is known as swirl sign. Acute hematoma may appear isodense to gray matter in patients of severe anemia. Subacute hematoma has a hypodense rim surrounding hyperdense hematoma and may show ring enhancement on post-contrast images reflecting the breach in blood brain barrier. Chronic hematoma is hypodense to gray matter.

\section{Non-contrast MRI}

It is used as a problem solving tool in patients who are stable and whose clinical features are not explainable by CT findings. On T1W and $\mathrm{T} 2 \mathrm{~W}$ images, blood appears hyperintense in subacute stage, hypointense in acute and is to hypointense in hyperacute and chronic states. Certain sequences of image are particularly useful.

FLAIR (Fluid attenuated Inversion Recovery) images are very sensitive in conspicuously delineating the gray and white matter lesions including DAI as well as acute and subacute subarachnoid hemorrhage ( $\mathrm{SAH})$ as hyperintense foci/areas. The only limitation is artefactual hyperintensity in sulci and cisterns in ventilated patients on high fraction of inspired oxygen (>0.6) [6].

Gradient recalled echo images (GRE) are very sensitive in the detection of blood products (acute to chronic) and hence conspicuously detect tiny hemorrhagic foci as a signal drop/ hypointense lesions. The major disadvantage is the susceptibility artefact in the region of paranasal sinuses and mastoid air cells limiting its role in detected cortical contusions in the inferior frontal and temporal lobes.

Fast low angle shot images (FLASH) that are recent advancement are more sensitive than GRE images in detecting fine hemorrhagic foci as in diffuse axonal injury with an advantage of higher spatial resolution [7]. This sequence is also known as "susceptibility weighted imaging (SWI)". SWI not only provide magnitude but also in-phase image out-phase images allowing differentiation between microcalcification image microhemorrhages.
Diffusion weighted images (DWI) detects areas of restricted diffusion of water molecules in tissues as hyperintense areas. As hemorrhagic areas have low diffusion coefficient, they appear as corresponding hypointense areas on apparent diffusion coefficient images (ADC). DWI and ADC images are more sensitive in detecting shearing injuries in white matter as compared to T2W, FLAIR and GRE images but are less sensitive in detecting hemorrhages than GRE images [8].

Diffusion tensor images (DTI) are directionally color coded images of the white matter tracts generated on the principle that water diffuses maximally in the direction parallel to the direction of tracts rather than in the direction perpendicular to them. These images can be, therefore utilized to assess the integrity of white matter tracts to determine the long term prognosis in CCT cases [9].

Proton magnetic resonance spectroscopy images (H1-MRS) detects the relative proportion of important primary metabolites of brain tissue in a voxel or a given volume of brain tissue. Reduction in the $\mathrm{NAA} / \mathrm{Cr}$ ratio and raised lactate levels are an indicator of tissue injury, and can be seen in patient with normal appearing brain on CT and conventional MRI [9]. Fall in NAA/Cr ratio is also seen in posttraumatic gliosis.

A correlation exists between low NAA image high choline levels and severity of brain injury. The latter is measured either in terms of GCS or length of post-traumatic amnesia [10].

Functional magnetic resonance images (fMRI) reflect the brain activity by utilizing the alterations in the ratio of deoxyhemoglobin to oxyhemoglobin in the cerebral blood. It can be used to study the posttraumatic neurological deficits. The role of this technique in CCT is still in the early phase.

\section{Single photon emission computed tomography (SPECT)}

It depicts the perfusion map of the brain in the form of color coded images. The technique involves the brain imaging by a gamma camera, two hours after intravenous tracer administration. The normal pattern is a symmetric tracer distribution with higher activity in occipital, temporal and parietal lobes; basal ganglia, thalami and cingulate gyrus.

These images correlate well with clinical status of the patient with acute CCT, better than acquired morphological images [11]. Normal brain SPECT examination is a very sensitive way of excluding any significant sequelae to CCT.

\section{Positron emission imaging/PET-CT}

Like SPECT, this modality aims at studying the cerebral perfusion based on the cerebral utilization of glucose by using 18-fluoro-dexyglucose (FDG) as tracer. Severe acute cerebral injury is associated with increased glucose utilization, decreased oxidative metabolism and uncoupling of cerebral blood flow.

Recent study has shown that PET images are more sensitive in detecting cerebral injury than conventional MRI especially those with persistent disturbance in behavior or cognition [12]. The major technical disadvantage of PET is low spatial resolution. The coupling of PET with CT i.e. PET-CT has overcome this limitation making it one of the most sensitive ways of determining the alterations in brain perfusion following CCT with its precise anatomical location. 


\section{Imaging Findings in Intra-Axial Injuries}

\section{Cerebral edema}

It arises as a result of loss of auto regulatory mechanism of cerebral vasculature to control cerebral perfusion as a response to parenchymal trauma especially in younger individuals. In the early stage, it is characterized by focal or diffuse parenchymal swelling or edema causing effacement of sulcal spaces, fissures, cisterns and ventricles with relative preservation of grey white matter differentiation. Later stages are characterized by progressive loss of gray white matter differentiation; sometimes producing white or reverse cerebellar sign characterized by diffusely hypodense cerebral hemispheres with relatively hyperdense cerebellum (Figure 1).

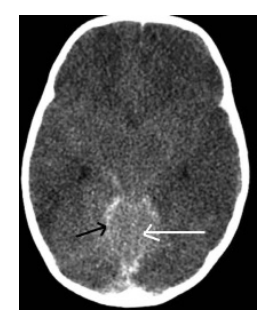

Figure 1: Non-contrast transaxial CT image shows diffusely hypodense cerebral hemispheres with complete loss of gray white matter differentiation and effacement of cisterns, sulcal space, fissures and ventricular system in a patient of trauma. Coexisting acute $\mathrm{SAH}$ is seen in the quadrigemial cisterns and along the sinus (black arrow). Superior part of cerebellum seen partially appears hyperdense (white arrow)-white cerebellar sign.

\section{Cortical contusions}

Classically, it appears as a small, hemorrhagic, wedge-shaped lesion with its apex directed centrally and base reaching up to the surface, usually located in the superficial cortex or peripheral gyri abutting the calvaria. Such lesions most commonly arise adjacent to orbital roofs, petrous and sphenoid ridges.

But when the impact is high, they become larger and rounded or irregular. MRI is the most sensitive technique for detection of cortical contusions as it is not limited by beam hardening or motion artefacts arising from calvarium that makes their recognition, sometimes difficult on CT. Besides this, multiplanar imaging of MRI compensates for the volume averaging artefacts.

\section{Intra Cerebral Hematoma (ICH)}

These are hemorrhagic collections in the deep brain parenchyma. Sometimes, they are not present immediately after traumas but appear over a period of 24-72 hours following injury. They usually appear in previously hypodense or ischemic areas possibly reflecting reperfusion injury (Figure 2).

\section{Subcortical gray matter injury}

It refers to the petechial or larger hemorrhagic lesions occurring in the deep gray matter including the thalamus, basal ganglia and upper brainstem [13]. This is an uncommon type of injury seen in severe CCT patients and is associated with poor outcome (Figures 3 and 4).

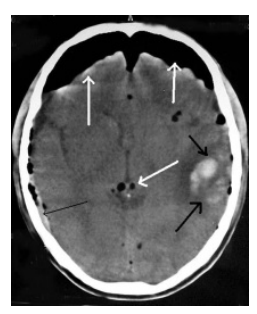

Figure 2: Non-contrast transaxial CT image shows cluster of cortical contusion in the left temporoparietal region (thick black arrows). Coexisting thin acute SDH is seen along the right lateral convexity (thin black arrow). Extra-axial collection of air is seen in the bilateral frontal regions anteriorly along with numerous aeroceles within the parenchyma (white arrows).

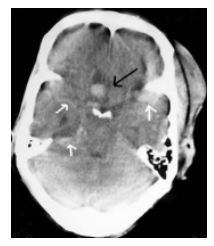

Figure 3: Non-contrast transaxial CT image shows a subacute ICH in the right basifrontal lobe in paramidline location posteromedially (black arrow). Coexisting SAH is seen in the basal cisterns and along left sylvian fissure (white arrows).

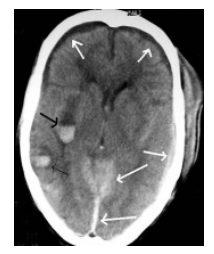

Figure 4: Non-contrast transaxial CT image shows subacute hemorrhage in the right putamen (thick black arrow). Coexisting $\mathrm{SDH}$ in different stages of evolution is seen-acute along tentorium, interhemispheric and left lateral convexity and subacute along bilateral frontal regions (white arrows). Cortical contusion is also seen in the right temporal lobe posterolaterally (thin black arrow).

\section{Diffuse axonal injury (DAI)}

It refers to the multiple, ovoid to ellipsoid, usually subcentimeter hemorrhagic $(20 \%)$ or non-hemorrhagic $(80 \%)$ lesions scattered diffuse in the white matter arising secondary to shearing white matter injury most commonly involving the frontal parasagittal white matter, splenium of corpus callosum and dorsolateral brainstem constituting the shearing injury triad. Commonest location is gray and white matter junction. CT is not very sensitive in the detection of DAI due to presence of beam hardening and volume averaging artefacts especially in the brainstem with frequent discordance between the clinical status of patient and CT images. MRI especially conventional spin-echo, GRE, SWI and DWI images are very sensitive in the detection of DAI. Although the treatment of these lesions are non-surgical, but they are 
more significant in determining the post-traumatic prognosis (Figures 5 and 6).

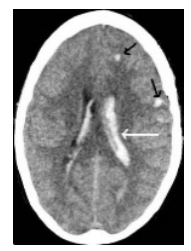

Figure 5: Non-contrast transaxial CT image shows small hemorrhagic lesions in the white matter (both deep and subcortical) in left cerebral hemisphere (black arrows). Coexisting IVH is seen especially in the body of left lateral ventricle (white arrows).

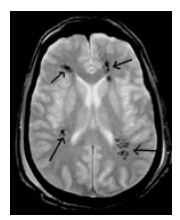

Figure 6: Transaxial GRE MR image shows multiple clusters of signal drop in the periventricular white matter in a patient of trauma suggestive of DAI (black arrows).

\section{Brainstem injuries}

These include primary traumatic lesions (contusion, DAI, pontomedullary rent or separation) and secondary lesions (hypoxicischemic injury and Duret's hemorrhage). Duret's hemorrhages are the result of injury to brainstem secondary to descending transtentorial herniation. In contrast to contusions and DAI, these occur in the central location especially pons and are associated with a very poor outcome.

\section{Penetrating or perforating injuries}

These injuries are characterized by breach in calvarium and meninges. Technically, penetrating injuries have only entry wounds while perforating injuries have both entry and exit wounds. Most of these arise as a result of gunshot but may also arise as a result of sharp and powerful blow to head by knives, nails, screwdrivers and sometimes in motor vehicle accidents. Three mechanisms are responsible for trauma-laceration/crushing, cutting or cavitation and shock waves [14]. Neuroimaging findings that affect management and prognosis of patients with penetrating injuries include the location of entry image exit wound; presence of intracranial foreign bodies; missile trajectory; presence of pneumocephalus; transventricular or trans-midline involvement; number of lobes involved; presence of cerebral edema and midline shift [15]. CT is, thus, the modality of choice in cases of penetrating especially gunshot injuries as it can reveal the presence, number and location of pellets or bullet or any other foreign body including bone [15]. The trajectory can be well visualized by CT [16]. In cases, where the bullet has passed through the skull, beveling is seen at the inner table at the entry site and at the outer table at the exit site. There is more extensive bone loss at the exit site. High velocity missile injuries are more severe and may even occur far away from the trajectory due to the radially spreading shock waves. Penetrating or perforating injuries are frequently complicated by extraaxial injuries, vascular injuries and presence of bone or soft tissues within the brain parenchyma leading to secondary infections and poor prognosis. Plain film radiography and fluoroscopy are however, more accurate in determining the size of projectile than $\mathrm{CT}$ as the presence of beam hardening artefacts limits the estimation of size and exact shape (Figures 7 and 8).

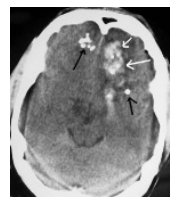

Figure 7: Noncontrast transaxial CT image shows multiple lead pellets in bilateral basifrontal lobes (black arrows) with hemorrhage in the adjacent parenchyma (white arrows).

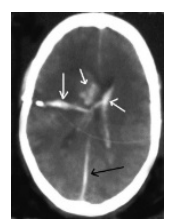

Figure 8: Non-contrast transaxial CT image with wide window settings shows hemorrhage along the trajectory of missile (large white arrow). Coexisting IVH (small white arrows) and interhemispheric acute SDH (black arrow) are also seen.

Patient suspected with vascular injuries are evaluated by angiography to rule out traumatic dissections, pseudoaneurysm, arteriovenous fistula and dural sinus laceration. Risk of vascular injury is high, if the trajectory passes adjacent to or through sylvian fissures; supraclinoid carotid, cavernous sinuses or major venous sinuses.

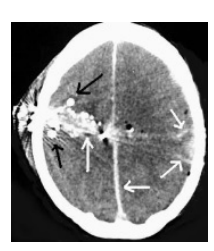

Figure 9: Non-contrast transaxial CT image shows hemorrhage (large white arrow) along the trajectory of missile with few showing beam hardening artefacts (black arrows). The trajectory is seen to cross the midline with hemorrhage in the left frontoparietal region laterally along with acute interhemispheric SDH (small white arrows).

Presence of significant unexplained subarachnoid hemorrhage or delayed hematoma should also arouse suspicion of vascular injury. CT angiography is the preferred technique. MR angiography should not be used unless presence of metallic foreign body has been ruled out by prior CT scan. MRI is particularly useful in the detection of wooden or glass fragments within the brain. Plain radiographs are indicated only when the CT is unavailable (Figure 9). 
Page 5 of 8

Important complications of penetrating/perforating injuries include infections (osteomyelitis, epidural abscess, subdural empyema, meningitis, brain abscess), post-traumatic epilepsy, CSF leaks (acutewithin 1 week and delayed-after 1 week), cranial nerve deficits, pseudoaneurysms and AVM [14]. Both CT and MRI can be used to evaluate them.

\section{Imaging Findings in Extra-axial Injuries}

\section{Fractures}

$\mathrm{CT}$ is a very sensitive technique in the detection of all type of fractures. Complex fractures can be studied well using 3D imaging with subsequent multiplanar reconstructions. Scout film of skull should be given careful attention to detect horizontal and thin vertical fractures. Scout film obviates the need for skull radiographs. Presence of air-fluid levels must be noted in the sinuses, mastoid air cells and middle ear cavity as they are indirect evidences of fractures of corresponding bones (Figures 10 and 11).

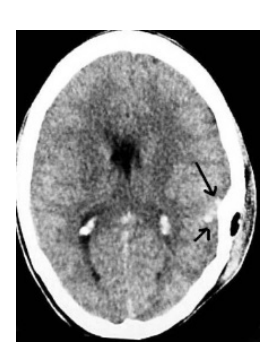

Figure 10: Non-contrast transaxial CT image shows cortical contusions in the left parietal lobe anterolaterally (black arrows) due to the overlying depressed fracture of parietal bone.

Presence of aeroceles is a sensitive indicator of fracture involving the sinuses or mastoid in the absence of compound fracture of skull. Thinner slices (1-2 mm) are indicated whenever skull base fractures are suspected. Presence of air in the temporo-mandibular joint in a patient of trauma is a sensitive indicator of ipsilateral temporal bone trauma. All fracture sites should be assessed for the presence of overlying scalp laceration and any foreign body to minimize the risk of subsequent infection.

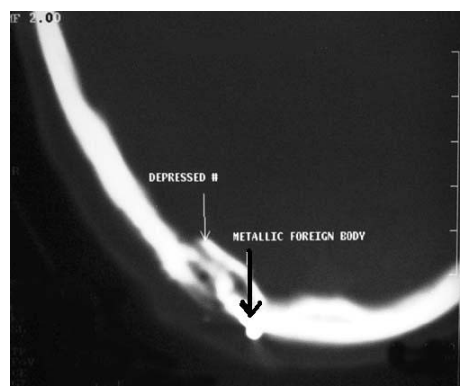

Figure 11: Noncontrast transaxial CT image shows comminuted depressed fracture of the occipital bone on right side (white arrow) caused by high impact by missile; one of the pellet is found impacted in the soft tissues of scalp (black arrow).
Depressed fractures need extra-attention, especially if they overlie motor cortex or venous sinus and when dural tear or intrusion of bone in to brain parenchyma are associated. Diastatic fractures are diagnosed when the separation of suture exceeds $3 \mathrm{~mm}$. Presence of otorrhea or rhinorrhea should arouse the suspicion of basal fracture of skull.

In all cases of suspected trauma, the visualized cervical spine, sinuses and orbits should be examined both in axial and 3D MPR as well as in scout film. In cases of any suspicious fracture that requires surgical management, additional scanning should be done at the same time to avoid time loss and need for shifting the patient again.

\section{Subdural hematoma (SDH)}

Classically, it presents as a crescentic or concavo-convex collection opposite the site of impact or fracture (contrecoup lesion) secondary to the tearing of the veins bridging the subdural space. It can cross the sutures except the sagittal suture and hence cannot cross the midline. It cannot extend from supratentorial to infratentorial compartment; however, it can spread along the falx or tentorium cerebelli. It is associated with a high incidence of underlying parenchymal injury.

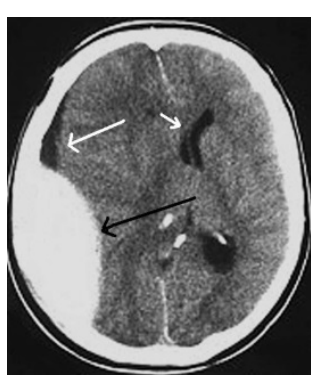

Figure 12: Non-contrast transaxial CT image shows typical acute EDH along right parietal convexity (black arrow) with associated subdural hygroma (large white arrow) and subfalcine herniation of right lateral ventricle to contralateral side along with midline shift (small white arrow).

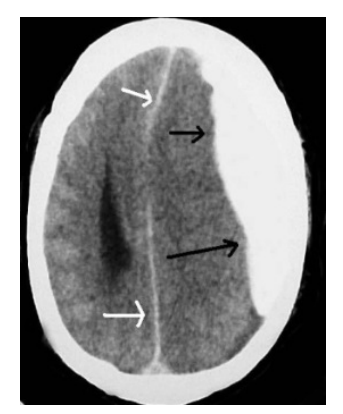

Figure 13: Non-contrast transaxial CT image shows typical acute SDH along left lateral convexity (black arrows) with acute interhemispheric SDH and midline shift to right side (white arrows).

Heterogeneity within an acute SDH may be due to unclotted blood or CSF admixture due to arachnoid laceration. Chronic SDH on the other hand shows fluid-fluid levels, loculations and septations. CT has 
a limitation in identifying thin and subacute (isodense) subdural hematoma. These limitations can be minimized by changing the window settings with wide window width and identifying secondary signs as midline shift and white matter buckling. Contrast administration can identify the bridging cortical veins and enhancing dura and arachnoid enveloping the collection in chronic cases. SDH as thin as 1-2 $\mathrm{mm}$ are missed by CT but they are clinically insignificant [13]. MRI can identify them easily and hence are used in doubtful cases (Figures 12-15).

\section{Extradural/Epidural hematoma (EDH)}

It is classically seen as a lenticular or biconvex collection at the skull convexities underlying the fracture (coup lesion) especially the temporal squamosa. These may have arterial or venous origin. The former in contrast to the latter have the propensity of expansion and subsequent mass effect. They are limited by sutures except sagittal suture and can cross the midline. It can extend from the supratentorial to infratentorial compartment. Presence of hypointense intact lining between the collection and brain surface on MRI is diagnostic. Similarly, displacement of the venous sinuses indicates the extradural location. Arterial type occurs in the supratentorium while venous type is commoner in posterior fossa.

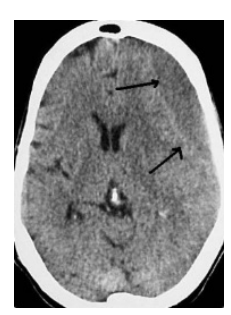

Figure 14: Non-contrast transaxial CT image shows typical chronic SDH along left lateral convexity anteriorly (black arrows) with effacement of sulcal spaces and mass effect over the ipsilateral lateral ventricle.

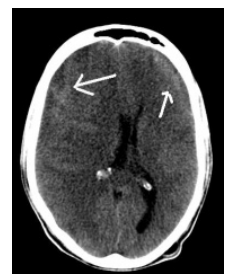

Figure 15: Non-contrast transaxial CT image shows typical bilateral acute on chronic SDH along lateral convexities anteriorly (white arrows) with mass effect causing a midline shift towards left side.

\section{Subdural hygroma}

It appears as a subdural collection of CSF attenuation usually occurring 72 hours after CCT. These are secondary to one-way rent in the arachnoid mater. Its distinction from chronic SDH may be difficult in the absence of any other sign of acute injury. MRI is very sensitive in identifying old degradation products of blood and is invaluable in differentiating subdural hygroma from chronic SDH. Subdural hygroma follows CSF signal intensity on all sequences.

\section{Subarachnoid hemorrhage (SAH)}

Acute SAH is seen on CT as linear hyperdensities within the sulcal space, fissures and cisterns usually opposite to the site of fracture or impact (contrecoup injury). The most common location of traumatic $\mathrm{SAH}$ is sylvian fissures or interpeduncular cisterns. Subacute or chronic SAH can be easily missed on CT but MRI with FLAIR, GRE or SWI sequences can identify it. Resolving SAH can interfere with CSF drainage from arachnoid granulations and hence can result in communicating hydrocephalous (Figures 16-18).

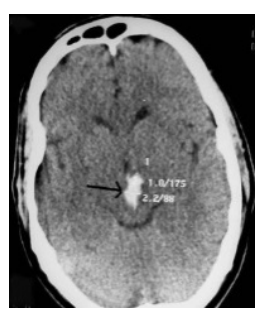

Figure 16: Non-contrast transaxial CT image shows interpeduncular acute SAH (black arrow) in a patient of trauma.

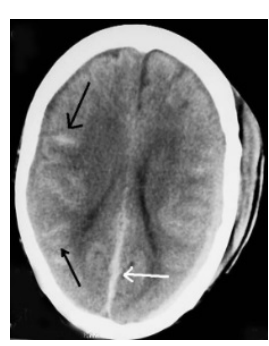

Figure 17: Non-contrast transaxial CT image shows typical acute sulcal bleed in the bilateral frontoparietal region; more prominent on right side (black arrows). Coexisting acute posterior interhemispheric SDH is also noted (white arrow).

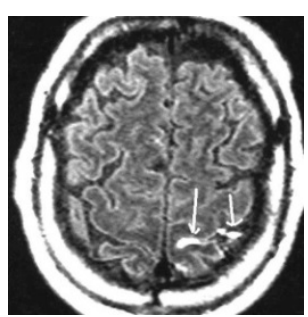

Figure 18: Transaxial FLAIR MR image shows acute sulcal SAH in the left parietal lobe near the vertex (white arrows).

\section{Intraventricular hemorrhage (IVH)}

It is seen as blood attenuating collection within the ventricular cavity. The most common appearance is the presence of fluid-blood level in the occipital horns of the lateral ventricle known as hematocrit effect. Sometimes tumefactive clots are seen as high attenuating solid lesions adherent to the choroid plexus or in the dependent part of occipital horns without layering of blood. Presence of blood in the 
fourth ventricle indicates poor prognosis. Presence of significant amount of blood in the ventricular system may impede CSF circulation resulting in communicating hydrocephalus (Figures 19 and 20).

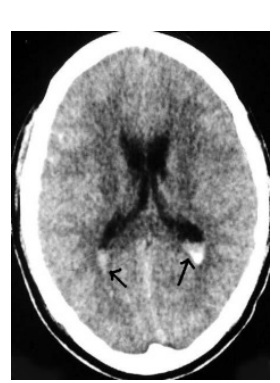

Figure 19: Non-contrast transaxial CT image shows CSF-blood level in the dependent part of the occipital horns of the bilateral lateral ventricle (black arrows) in a patient of trauma hematocrit effect.

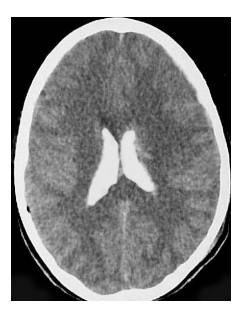

Figure 20: Non-contrast transaxial CT image shows hyperdense blood filling the body of the lateral ventricles on both sides in a patient of trauma.

\section{Complications Image Sequelae of CCT}

These can be broadly divided in to two categories:

\section{Early onset}

Delayed intracranial hemorrhage, diffuse cerebral edema, pneumocephalus, CSF leaks, cerebral ischemia, herniations (subfalcine, descending and ascending transtentorial, transalar and tonsillar types).

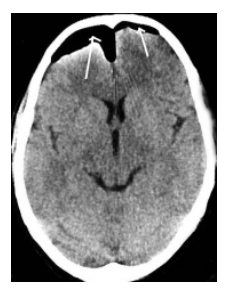

Figure 21: Non-contrast transaxial CT image shows extra-axial collection of air in the anterior frontal bilaterally $(R>>L)$ in a patient of trauma.

\section{Late onset}

Cerebral, cerebellar or corpus callosal atrophy, cerebral infarction or necrosis, cephaloceles image leptomeningeal cyst or growing fractures, post-traumatic arachnoid cyst, pseudoaneurysms, arteriovenous fistulas, arterial or venous sinus thrombosis and post-traumatic hypopituitarism leading to diabetes insipidus (Figures 21 and 22).

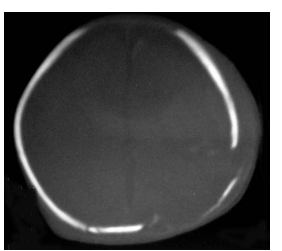

Figure 22: Non-contrast transaxial CT image shows growing fracture of left parietal bone; thinning of bone with brain matter bulging through the defect and pushing the bone.

\section{Non-accidental CCT in child (Child abuse)}

No discussion on CCT trauma is complete if neuroimaging of nonaccidental CCT in child is excluded. Imaging features of CCT in a case of child abuse or battered baby are characteristic.

- Fractures: Presence of multiple, complex or different type of fractures concomitantly without evidence of significant trauma should arouse the suspicion.

- Extra-axial hematoma: Subdural hematoma in different stages of evolution is characteristic for child abuse. They may also be seen along the inter hemispheric fissure

- Cortical contusions and DAI

- Cerebral edema and ischemia: Diffuse cerebral edema is a common manifestation of child abuse from 0-4 years of age. Global cerebral ischemia may be seen secondary to attempts of strangulation or smothering.

\section{Conclusion}

Noncontrast CT is modality of choice and probably the only examination needed in the setting of acute CCT in majority of the cases. The primary role of CT in such cases is to identify lesions that might require immediate neurosurgical intervention. Besides, the intracranial lesions, CT is very sensitive in detecting fractures of skull base and complex fractures of face and spine. CT is fast, easily available, relatively cost-effective and compatible with various life support systems. Role of MRI is reserved as a problem solving tool especially in cases of unexplainable brain dysfunction following CCT and in subacute image chronic cases. MRI imaging is indicated sometime within first two weeks of CCT in patients with moderate to severe injuries $[13,17]$. Skull films are only useful in identifying linear fractures. Use of advanced neuroimaging using MRI, SPECT and PET is reserved for chronic cases with long term neurological disabilities.

\section{References}

1. MacDorman MF, Minino AM, Strobino DM, Guyer B (2002) Annual summary of vital statistics: 2001. Pediatrics 110: 1037-1052.

2. Lee B, Newberg A (2005) Neuroimaging in traumatic brain imaging NeuroRx 2: 372-383.

3. Haydel MJ, Preston CA, Mills TJ, Luber S, Blaudeau E, et al. (2000) Indications for computed tomography in patients with minor head injury. N Engl J Med 343: 100-105. 
Citation: $\quad$ Rastogi R, Bhargava S, Joon P, Gupta Y, Wani AM, et al. (2016) Neuroimaging in Craniocerebral Trauma. Brain Disord The 5: 218. doi:

Page 8 of 8

4. Gean AD (1994) Imaging of Head Trauma. New York: LippincottWilliams imageWilkins.

5. Sosin DM, Sniezek JE, Thurman DJ (1996) Incidence of mild and moderate brain injury in the United States, 1991. Brain Inj 10: 47-54.

6. Frigon C, Jardine DS, Weinberger E, Heckbert SR, Shaw DW (2002) Fraction of inspired oxygen in relation to cerebrospinal fluid hyperintensity on FLAIR MR imaging of the brain in children and young adults undergoing anesthesia. AJR Am J Roentgenol 179: 791-796.

7. Tong KA, Ashwal S, Holshouser BA, Shutter LA, Herigault G, et al. (2003) Hemorrhagic shearing lesions in children and adolescents with posttraumatic diffuse axonal injury: improved detection and initial results. Radiology 227: 332-339.

8. Ezaki Y, Tsutsumi K, Morikawa M, Nagata I (2006) Role of Diffusion Weighted Magnetic Resonance Imaging in Diffuse Axonal Injury. Acta Radiol 47: 733-740.

9. Xiong KL, Zhu YS, Zhang WG (2014) Diffusion tensor imaging and magnetic resonance spectroscopy in traumatic brain injury: a review of recent literature. Brain Imaging Behav 8: 487-496.

10. Kubas B, Lebkowski W, Lebkowska U, Kułak W, Tarasow E, et al. (2010) Proton MR spectroscopy in mild traumatic brain injury. Pol J Radiol 75: 7-10.
11. Camargo EE (2001) Brain SPECT in neurology and psychiatry. J Nucl Med 42: 611-623.

12. McAllister TW, Sparling MB, Flashman LA, Saykin AJ (2001) Neuroimaging findings in mild traumatic brain injury. J Clin Exp Neuropsychol 23: 775-791.

13. Indrajit IK, Mohan C (2007) Imaging in head trauma - a review. Journal of Postgraduate Medical Education. Training and Research 2: 69-74

14. Vinas FC (2015) Penetrating head trauma. Medscape.

15. Kazim SF, Shamim MS, Tahir MZ, Enam SA, Waheed S (2011) Management of penetrating brain injury. J Emerg Trauma Shock 4: 395-402.

16. Hollerman JJ, Fackler ML, Coldwell DM, Ben-Menachem Y (1990) Gunshot wounds: 1. Bullets, ballistics, and mechanisms of injury. AJR Am J Roentgenol 155: 685-690.

17. Ruth G, Ramsey, Scheer-Williams M (2006) Neuroradiologic evaluation of craniocerebral trauma. Behavioral Sciences and the Law 5: 239-285. 